\title{
Large Interstellar and Cometary Biomolecules
}

\author{
Lewis E. Snyder \\ Department of Astronomy, University of Illinois, 1002 W. Green, \\ Urbana, Illinois 61801, USA
}

\begin{abstract}
The relevance of hot molecular cores (HMCs) to the solar nebula has been strengthened as evidence emerges for a hot core phase in low mass star formation regions. Consequently, interferometric studies of large highly saturated molecules of biological interest (biomolecules) in HMCs have been extended to comets. A key goal is the interferometric detection and mapping of large biomolecules, which could be formed along chemical pathways similar to those for HMC species.
\end{abstract}

\section{Galactic Star Formation Regions and Hot Molecular Cores}

Much progress has been made toward understanding the formation of solartype stars by focusing on low-mass regions (Mundy, Looney, \& Welch 2000). However, the most interesting and complex chemistry of molecules of biological interest (biomolecules) has been observed in hot molecular cores (HMCs) mainly in regions of massive star formation. Complex saturated molecules have been reported for only one low-mass young stellar object, IRAS 16293-2422 (Cazaux et al. 2003). Because of their relatively large sizes and column densities, highmass HMCs provide an environment in which the chemical pathways for many biomolecules can be directly inferred from observations. The chemistry in HMCs is more complex than that detected so far in the cold dark clouds, which have been the targets of most low-mass studies. Large, highly saturated molecules have been mostly detected in HMCs. Examples include ethyl cyanide, methyl formate, methanol, dimethyl ether, acetone, glycolaldehyde, and acetic acid. The high temperatures in HMCs permit a chemistry to take place that ordinarily would not occur in cold dark clouds (Ehrenfreund \& Charnley 2000). The rich, active chemistry of HMCs is confined to small regions near the heating sources, and so molecular detections are enhanced by telescopes that have high spatial resolution, such as interferometric arrays. There are many gaps in our knowledge about the evolutionary progression from a high-mass HMC to a primordial solar nebula. For example, detailed data are needed for HMC sizes, masses, structures, kinematics, and temperatures. Much of this can be obtained through high spatial resolution studies of large, highly saturated molecules in HMCs. Coincidentally, many of these large species are also of biological interest, which makes the potential connection to the solar nebula even more interesting. 


\section{Current Research on Large Molecules in HMCs}

Over the last few years, interferometric array measurements have revealed a hot core of unusually high column density embedded within the more extended molecular cloud in Sgr B2(N). It is called the Large Molecule Heimat source, or Sgr B2(N-LMH), due to its extraordinarily high column densities of large, highly saturated molecules, including many of pre-biotic interest (Snyder et al. 1994; Miao \& Snyder 1997). The interferometric array measurements were made with synthesized beams that ranged from $1^{\prime \prime}$ to $14^{\prime \prime}$ in width. Given the distance to Sgr B2 of $7.1 \mathrm{kpc}$ (Reid 1993), an angular resolution of $1^{\prime \prime}$ corresponds to a physical scale of $\sim 7500 \mathrm{AU}$, or $\sim$ one-tenth the size of the Oort cometary cloud. A consistent set of beam-averaged column densities measured with the BIMA Array, all at a few arcseconds angular resolution, has been summarized by Snyder et al. (2002) for several large dust generated molecular species in Sgr B2(N-LMH): $2.9(3) \times 10^{16} \mathrm{~cm}^{-2}$ for acetone; $0.6(1) \times 10^{16} \mathrm{~cm}^{-2}$ for acetic acid; $1.1(3) \times 10^{16} \mathrm{~cm}^{-2}$ for formic acid; $4.6(1) \times 10^{16} \mathrm{~cm}^{-2}$ for ethyl cyanide; and $11.2(10) \times 10^{16} \mathrm{~cm}^{-2}$ for methyl formate. Note the relatively high column density of acetic acid. Acetic acid is of possible pre-biotic importance because its structure is only separated by an $\mathrm{NH}_{2}$ fragment from glycine, the simplest amino acid. In Sgr B2(N-LMH), its column density is $\sim 20$ times less than that of its isomer, methyl formate. This suggests that large molecules in the pre-solar nebula are not formed from randomly assembled constituent atoms.

Because it is important to learn how well the astrochemistry found in Sgr B2(N-LMH) really represents the astrochemistry of HMCs in the disk of the Galaxy, searches for new sources of large molecules outside the galactic center region have been conducted by Remijan et al. (2002; 2003). As a result, acetic acid has been detected in the high-mass sources W51e2 and G34.3+0.2. In the 3 known high-mass sources, the acetic acid column density appears to be $\sim 15-100$ times below the abundance of its isomer methyl formate, $\sim 2-10$ times below the abundance of formic acid, and $\sim 2-10$ times below the abundance of ethyl cyanide. (Cazaux et al. (2003) detected only one line of acetic acid in IRAS 16293-2422, which makes the relative abundance determination difficult in this source.)

\section{Comets}

Bockelée-Morvan et al. (2000) conducted an extensive study of Comet C/1995 O1 (Hale-Bopp) with the Caltech Submillimeter Observatory, the IRAM 30 m, and the Plateau-de-Bure interferometer, all in autocorrelation mode. They observed 16 molecular species, including 3 which are likely to be made either abundantly or exclusively by grain chemistry: formaldehyde, methanol, and methyl formate $(\mathrm{MeF})$. The detection of methyl formate provides the first clear chemical link between the chemistry of comets and that of biomolecules observed in highmass hot molecular cores. Bockelée-Morvan et al. (2000) calculated a molecular abundance relative to $\mathrm{H}_{2} \mathrm{O}$ of $0.08 \%$ for methyl formate and $\mathrm{Q}(\mathrm{MeF})=2.8 \times$ $10^{28} \mathrm{~s}^{-1}$. The upper limits from BIMA Array observations are more than 3 times higher. If the same molecular formation chemistry active in HMCs applies to Comet Hale-Bopp, to find acetic acid (AcA) it would have been necessary to 
reach $\mathrm{Q}(\mathrm{AcA})=(3-20) \times 10^{26} \mathrm{~s}^{-1}$. The BIMA Array limit for Hale-Bopp at $3 \mathrm{~mm}$ wavelength was $5 \times 10^{28} \mathrm{~s}^{-1}$, or 25 times greater than the best possible case for detecting acetic acid (Friedel et al. 2005).

\section{Conclusions}

HMCs in high-mass star formation regions produce large biomolecules which now are starting to be found in HMCs in low-mass regions. Among these large biomolecules, only methyl formate has been found in a comet (Hale-Bopp). However, if the chemistry of comets follows the chemistry of HMCs, methyl formate may provide a connection to acetic acid and even to glycine in comets. The BIMA Array results from Hale-Bopp miss the sensitivity required to image cometary methyl formate by at least a factor of 3 . This situation will change with the construction of the Combined Array for Research in Millimeter-wave Astronomy (CARMA) at a new high site near Owens Valley Observatory in California. At wavelengths near $1 \mathrm{~mm}$, there will be a gain of at least an order of magnitude in sensitivity, which should introduce a new era in the imaging of large cometary biomolecules.

Acknowledgments. We acknowledge support from the Laboratory for Astronomical Imaging at the University of Illinois, NSF AST 99-81363, NSF AST 02-28953, and NASA NAG 5-4292.

\section{References}

Bockelée-Morvan, D., et al. 2000, A\&A, 353, 1101

Cazaux, S., et al. 2003, ApJ, 593, L51

Ehrenfreund, P., \& Charnley, S. B. 2000, ARA\&A, 38, 427

Friedel, D. N., et al. 2005, ApJ, 630, 623

Miao, Y., \& Snyder, L. E. 1997, ApJ, 480, L67

Mundy, L. G., Looney, L. W., \& Welch, W. J. 2000, in Protostars and Planets IV, eds. V. Mannings, A. Boss, \& S. Russell (U. Arizona Press), 355

Reid, M. J. 1993, ARA\&A, 31, 345

Remijan, A. J., Snyder, L. E., Fridel, D. N., Liu, S. Y., \& Shah, R. 2003, ApJ, 590, 314

Remijan, A., Snyder, L. E., Liu, S.-Y., Mehringer, D., \& Kuan, Y.-J. 2002, ApJ, 576, 264

Snyder, L. E., Kuan, Y.-J., \& Miao, Y. 1994, in The Structure and Content of Molecular Clouds, ed. T. Wilson \& K. Johnston (Springer-Verlag), 187

Snyder, L. E., et al. 2002, ApJ, 578, 245 